\title{
Geological Modelling of Stratified Upper Austrian Gas Reservoirs
}

\author{
Griess B., Kreczy L.
}

Rohöl - Aufsuchungs A. G., Austria

\begin{abstract}
Copyright 1995, Steering Committee of the European IOR - Symposium.
This paper was presented at the 8th. European IOR - Symposium in Vienna, Austria, May 16 - 17, 1995

This paper was selected for presentation by the Steering Committee, following review of information contained in an abstract

submitted by the author(s). The paper, as presented has not been reviewed by the Steering Committee.
\end{abstract}

\begin{abstract}
The development of high capacity desktop computers (Workstations) has increased dramatically the possibilities of investigating stratified reservoir characteristics in greater detail than previously.

The geological modeling package SGM (Stratigraphic Geocellular Modeling) was used to build complex 3D reservoir models of several Upper Austrian gas reservoirs for two general purposes:
\end{abstract}

1. Stratified and at random distributed sand lenses of depleted gas reservoirs require detailed investigations of their potential as underground gas storage's for an increased demand in future.

2. For the same type of reservoirs with strong water drives, 3D - models were built for a better under standing of reservoir parameters and flow paths.

Both types of reservoirs have been modeled in detail (up to $1,500.000$ cells, vertical resolution of 1 foot) and later been condensed to appropriate complex $3 \mathrm{D}$ reservoir simulation models. The current reservoir simulation model of the underground gas storage Puchkirchen is based on a SGM model. The results of the simulation compare very well with recently observed production test data of horizontal wells.

The most important benefits from detailed reservoir characterizations are possibilities of predicting future storage behavior based on historical experience with a high degree of accuracy. For reservoirs to be used as new storage, these experiences can be utilized for a realistic and optimal design of surface facilities, that fit the reservoir parameters needs.

\section{Introduction}

Most of the gas reservoirs that are located in RAG's concession area of Upper Austria (Fig. 1) are not massive, but stratified and at random distributed sand lenses. It is a mature area and new opportunities have to be found e.g. to utilize depleted reservoirs as seasonal underground gas storages.

In the case of improving recovery more efforts are necessary for volumetric mapping of remaining reserves. Several reservoirs show extremely strong water drives, some of them indicating aquifer volumes, that hardly fit into the geological picture. Detailed characterization of the reservoir sands should help to get a better understanding of what is happening and thus lead to find new methods for an increased recovery.

Planning work for RAG's underground gas storage Puchkirchen has been assisted strongly by reservoir simulation. The necessary preparation of 3D geological models was done with Stratamodel's SGM. The software is capable of combining well log information with seismic -, or well marker depth maps, that form a stratigraphic framework. Especially for locating the Puchkirchen horizontal wells and planning of the well paths the model has proved very rewarding.

Installed workstation memory and disk space allow currently a vertical resolution of about 30 centimeters. The horizontal grid spacing is in the range of 100 meters. Thus also very thin pays of less than 50 centimeters can be mapped volumetrically. Interpolation for the main attribute's porosity and saturation can be made for the entire 3D - model. Mathematical operations allow the calculation of volumes for each tiny cell. Volumes can be summed up for the total or for separated layers or parts of the model.

The modeling software in combination with a powerful workstation allows to visualize different reservoir models and find the best possible view. Fast 


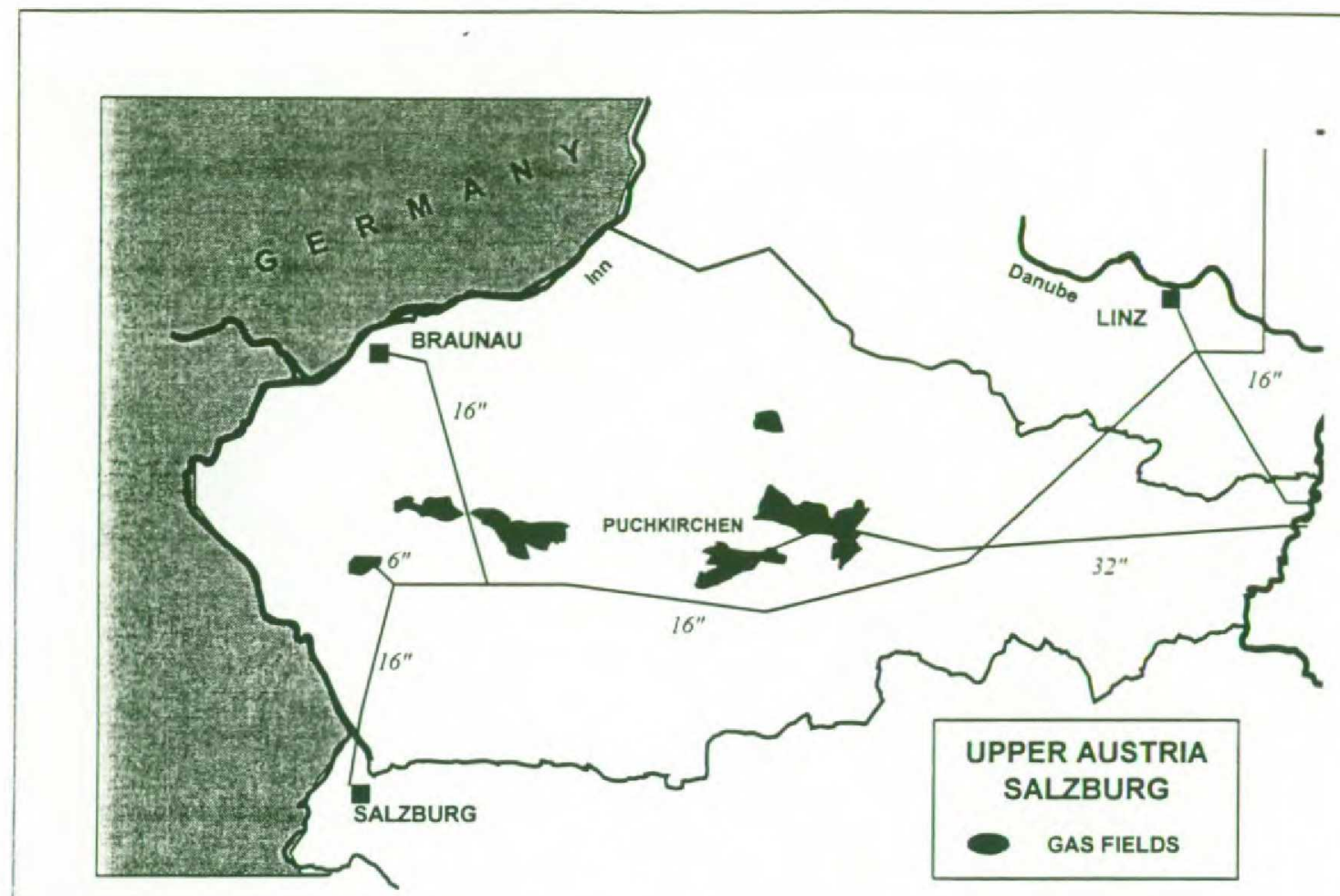

\section{Fig. 1 RAG Concession Area with Fields and Main Pipelines}

and detailed calculations of volumetrics assist in finding an acceptable reservoir model.

Interpolated and calculated attributes of the final fine gridded model can be condensed to dimensions that can be handled by a reservoir simulator. Finally the reservoir model is to be proved dynamically by history matching.

\section{Data}

In principle preparation of input data like structure grids for the stratigraphic framework model and well log data like porosity \& fluid saturation for the attribute model is very easy. The absence of a digital log and map database was a time consuming deficiency. Digitized log properties for all wells had to be prepared by hand. Due to stratification most of the well log data were prepared with a vertical resolution of 30 centimeters.

The stratigraphic framework for a reservoir is determined by at least one structure grid. Due to difficult log correlation in some of the reservoirs only a single top structure grid could be defined for building the model. Thus the model can only be built with constant cell thickness. Sand bodies, conglomerates and shales however never have constant thickness through out the reservoir. This will lead to conflicts when layers of the vertically fine SGM model have to be condensed to coarser simulation model layers, since the averaging can only be done within the same SGM model layers. However the model layers will probably not follow the geological layers.

In a first stage several reservoir models were built only using the top structure grid. Later improvements to the model can easily be done as it may be possible to define more markers in the complex formation and a more sophisticated framework model can be determined.

For the Underground Gas Storage (UGS) Puchkirchen several models have been built. The current reservoir simulation model is based on two top structure maps, the Upper Puchkirchen Formation (UPF) A 1, that has been used as a storage since 1982 and, separated by a $10 \mathrm{~m}$ tight shale, the lower A2, that watered out in the early eighties. The two structure maps determine two sequence boundaries (Fig. 2) of the petrophysical input data. The data for the attribute model came from 29 wells, located in the model area. Porosity data were mainly taken from logs and for five wells also from core measurements. Due to the history of field development (1970 to 1990) data was not available in the same density and quality for all wells. Some early achieved log porosity's were adapted to data of later drilled wells near by.

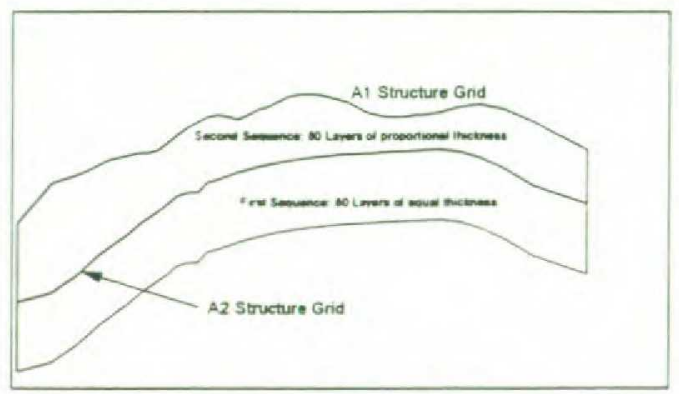

Fig. 2. Stratigraphic Framework Model of Puchikirchen UPF A1 \& A2 


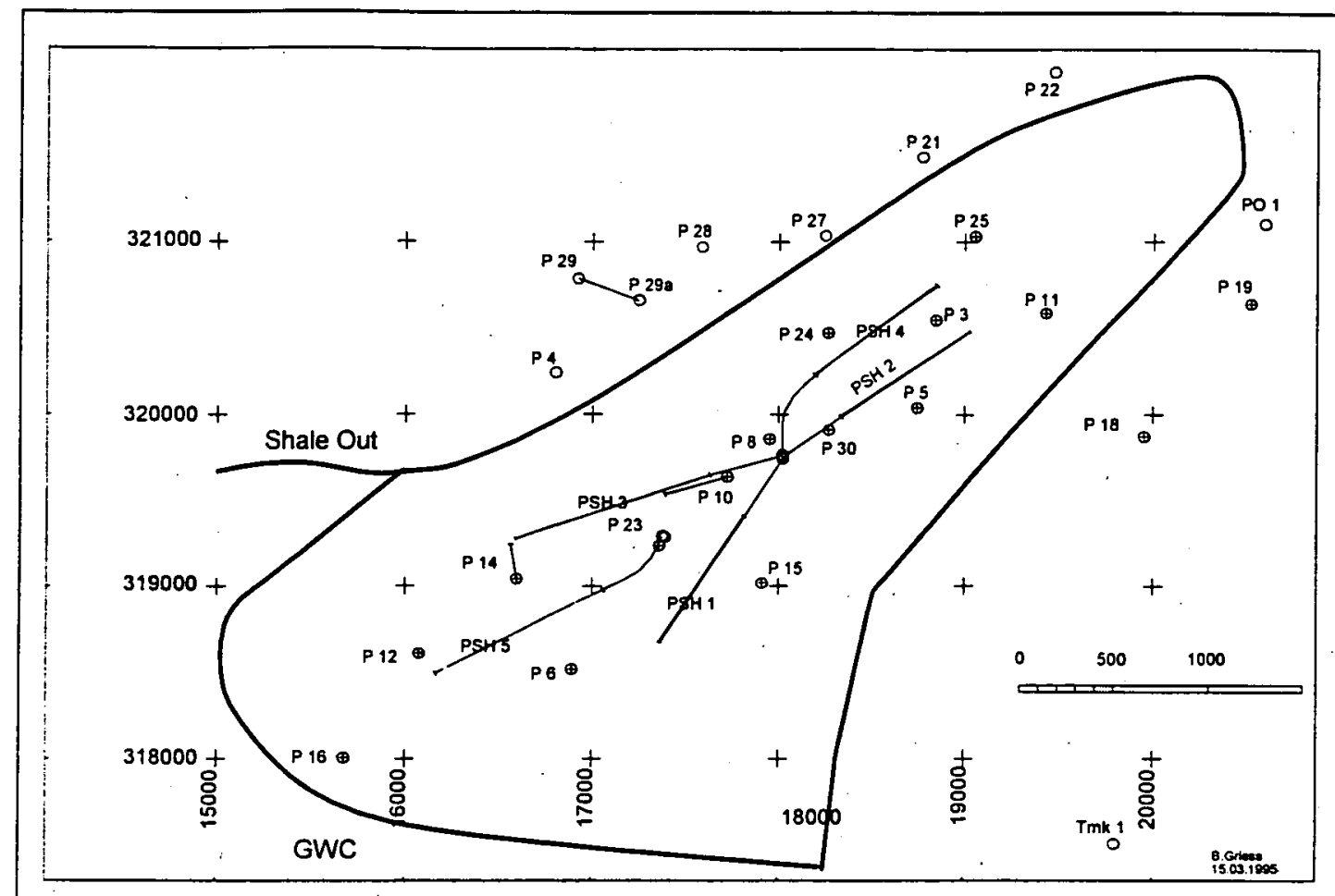

Fig. 3. Well Locations of Puchkirchen Gas Storage

Water saturation from logs was used as a second attribute. Data from watered out wells, had to be corrected with help of core measurement correlation of porosity, permeability and connate water saturations. The lower (UPF A2) of the two geological sequences was built below the top depth of $A 2$ with 80 layers. There was no bottom boundary map available, which resulted in a sequence with equal cell thickness all over. The upper sequence (UPF Al) was determined by two vertical boundaries. This resulted in proportional thickness for the grid cells ( 80 layers) as the distance between the two structures varies.

A second SGM model was prepared for the purpose of getting exact control over the target depth when drilling the horizontal storage wells. The decision had been made to set the production casing about 10 meters above the storage formation to avoid cementing in the gas sands. Four geological markers in the overlaying Hall Formation had been mapped over the entire area and included in the model. The relative distance between markers and reservoir top was postulated to be constant. For each of the horizontal wells the location and vertical depth of the markers were determined from the model. The actual positions measured in the drilling phase deviated vertically only by a maximum of two meters.

In the Al four different sand layers could be identified with the Alc and the Ald as the main layers with good thickness and sufficient aerial extent. The sand layers are often separated by thin shale beds or tight sandstones. From a reservoir standpoint the subdivision is not necessary - the Al sand layers can be considered as one reservoir. However the development of the storage by horizontal wells made it necessary to investigate more in detail the optimum well path for a connection of sands in the shalier parts of the reservoir. Finally $\log$ data from the five horizontal wells have been included into a new SGM model, which will be used for future updates of the reservoir simulation.

\section{Puchkirchen UGS}

The Puchkirchen gas field is located approximately midway between the cities of Salzburg and Linz in Upper Austria. The field was discovered in 1969 and production started in 1972. In the year 1982 RAG started to operate an underground gas storage (Fig. 3) in the Upper Puchkirchen Formation (UPF) Al sand. The first reservoir simulation studies to investigate for a larger storage potential were started in 1976. New coarse models continued in 1990. In early 1993 a detailed simulation study of both reservoirs (A1 \& A2) followed with the objectives of optimizing the layout for the present $(=500 \mathrm{MMcbm})$ contract and secondly to investigate the upper potential of the storage. A contract for a total tum over volume (TOV) in Puchkirchen of $500 \mathrm{MMcbm}$ has been signed and the storage will start to operate on Ist July 1995.

The geological model was mapped using Stratamodel's SGM software with about 360.000 cells. It was condensed to a 17.000 cell simulation model. Only minor fine tuning was necessary to achieve an excellent history match for 10 years of production (1972 - 1981) and 12 years (Fig. 4) of storage cycles (1982 - 1993).

In June 1993 the drilling of the first horizontal well was finalized. The reservoir part of the planning had 
been strongly based on the 3D geological model, with extremely satisfying results for the first project of this

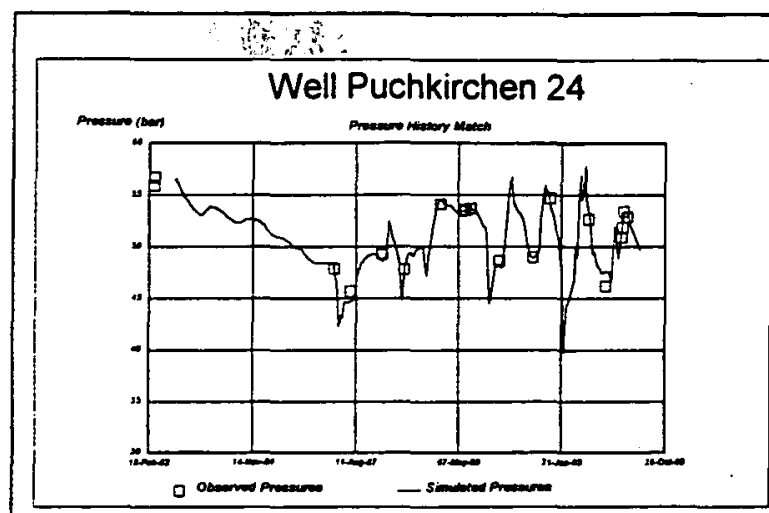

Fig. 4 Pertect Pressure Match of Storage Cycles

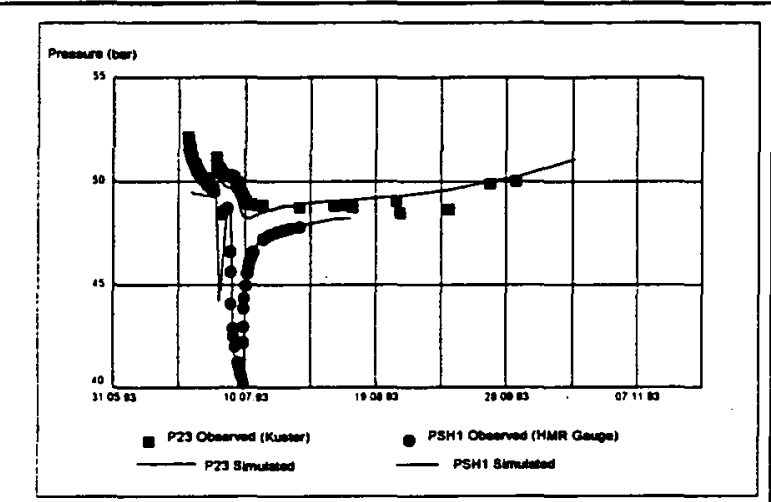

Fig. 5 Pressure Match of Horizontal Well Test

type in RAG. Based on the multilayer reservoir simulation model the capacity of the well had been very accurately predicted. Only minor changes were necessary to match a first production test.

Since June 1993 five horizontal: wells have been drilled. The results were very satisfying except for one of the wells, where the predicted capacity was only reached to about 80 percent. Because of MWD measurement problems the well path could not be optimally controlled and located within the target sand.

Production tests for four of the wells have been matched very precisely (Fig. 5). During two summer injection periods gas was injected into certain parts of the reservoir with simultaneous observation of the pressure changes in the other areas. Only minor fine tuning has been necessary to achieve an accurate pressure match. Figure 6 shows how successfully the first two horizontal wells were located in the relatively thin reservoir.

An observed pressure hysteresis between the early production phase and the historical $90 \mathrm{MMcbm}$ storage phase showed that a very accurate model would be necessary to predict storage scenarios for $500 \mathrm{MMcbm}$ or larger. The comparison of earlier coarse models with the final SGM based simulation model showed that at least one more horizontal well would be needed for the project.

In a short time from now (July 1995) the first storage injection cycle will start. Fig. 7 shows how the simulation model predicts the future storage behavior. The reservoir pressure in October 1995 will show how accurate our modeling has been.

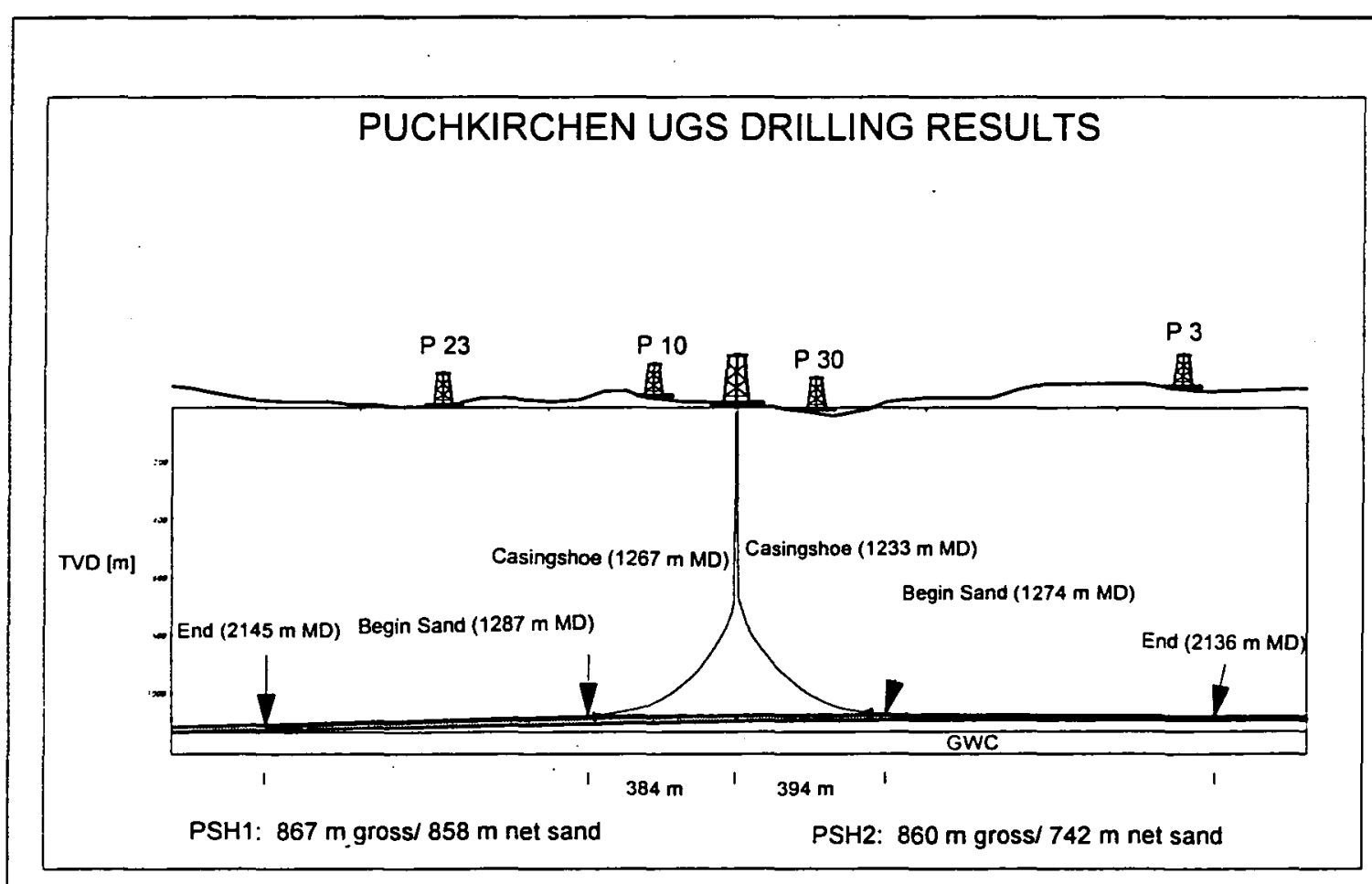

Fig. 6 Horizontal Well Drilling Results 


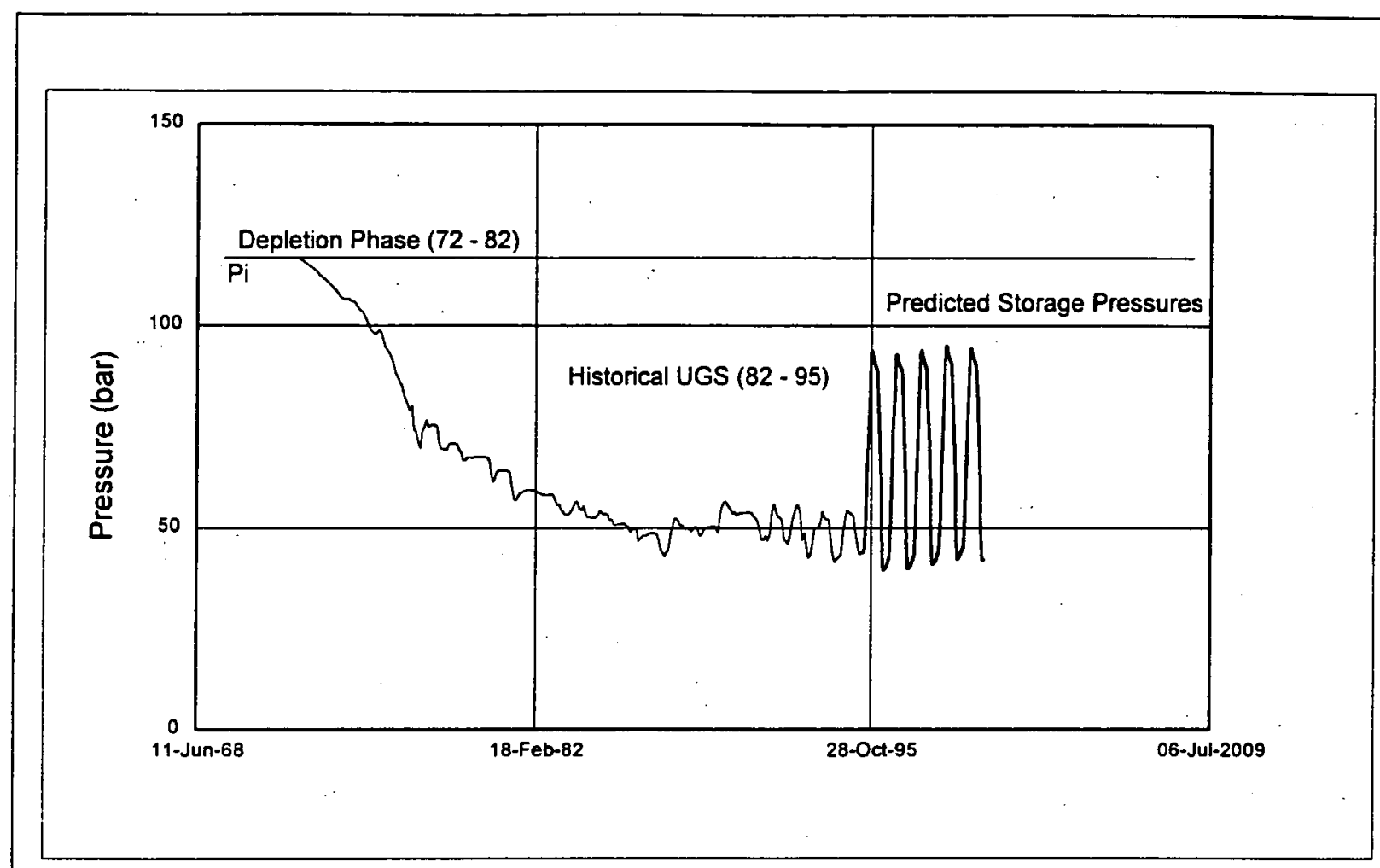

Fig. 7 Predicted Reservoir Pressures for TOV of $500 \mathrm{MMcbm}$

\section{Enhanced Gas Recovery}

In the last three years we have continuously increased the list of reservoirs being modeled in detail. In some cases SGM was only used as a new tool to achieve more accurate volumetric gas in place figures. It helped to either prove or change numbers, that had been achieved with other methods before.

Possibility of increasing demand for natural gas as an environmental friendly energy source in the future has lead to investigations of the potential for underground gas storage in other depleted Upper Austrian gas reservoirs.

The most important emphasis however is currently laid on the modeling of several gas reservoirs, that have a very strong water drive. Currently all wells are watered out, however the reservoir energy make us believe that their still could be some potential to increase recovery. The pressure decline after production of a substantial amount of the estimated gas in place was only a few bars. Material balance calculations did not really help. Volumetric calculations made in the past seemed to be uncertain.

Most of the water driven reservoirs are characterized by high permeability in good quality sands changing with tight conglomerates. Correlation of single sands within the complex reservoir is very difficult or almost impossible.

We think that there is only one possible way to model the complex geology of our strong water drive reservoirs. It is to build reservoir simulation models, that are as detailed as possible and thus the detailed description made by SGM is adequate. The objective of predicting trapped gas and increase recovery is very difficult to fulfill. The complex geology and the possibility to model all the single porous bodies let us face the problem of how to combine the very fine layers to appropriate simulation model layers. Other problems emerge with a very limited dataset for the permeability distribution through out the reservoirs.

The main result until today of detailed geological modeling has been more realistic numbers for initial gas in place than previously. We have now also more trust in the distribution of the gas in place.

A second important output have been multi cross sectional views. They help to understand the reservoir behavior and improve the model in combination with all other relevant information (DSTs, Core Analysis, Perforation Data, Production Data, Pressure Build Up Tests). The images of vertical and horizontal distribution of sands, shales and conglomerates can help to optimally locate new horizontal or slanted wells.

Several of RAG's volumetric reservoirs have first been simulated with simple 2D models. The later SGM based 3D models showed significant changes in the prediction of underground gas storage scenarios. In Puchkirchen the observations have proved, that the more complex reservoir models will give a more reliable prediction.

In most wells of the strong water drive reservoirs it was necessary to accelerate gas production in the past to avoid early water production and thus only very few pressure observations were made.

Currently ongoing reservoir simulation studies will show us whether satisfying results can be achieved with this limited amount of data. 


\section{Conclusions}

Although there still is room for improvements in our detailed geological modeling, it has given substantial contributions to RAG's most important project:

1. The SGM based reservoir simulation model of the underground gas storage Puchkirchen has been used now for two years to predict pressure behavior in possible storage scenarios. Based on the available reservoir history a very accurate match as the basis for prediction runs was achieved. Comparisons with coarser models and simple deliverability calculations well by well show us that previous results would have been to optimistic.

2. The detailed reservoir model has been of essential importance for the reservoir part of planning and drilling successfully five horizontal wells. Prediction of performance of the horizontal wells has been very close to reality.

3. Several other reservoirs have been modeled and condensed to appropriate simulation models in very short time and with a much greater detail than previously.

\section{Acknowledgments}

We thank RAG and RAG's shareholders Mobil Oil Corporation, Shell International and RBG for permission to publish this work. 